\title{
Prevention of Mental Illnesses Caused by Adverse Childhood Experience
}

\author{
Juezhe Huang \\ University of California, Davis, the United States \\ *Corresponding author. Email: Huangjuezhe.eric@gmail.com

\begin{abstract}
Adverse childhood experiences (ACEs) are potentially stressful events that children experience before the age of 18 years old. Numerous studies have linked negative health, as well as developmental and behavioral outcomes to the exposure of ACEs. This study analyzes existing research theories and results to discuss the relationship between ACE and mental health (such as depression, anxiety, and suicide). Findings provide support for a relationship between ACEs and the prospective risk of common mental illnesses. Some measures (Trauma informed care, Nurse-Family Partnership) may reduce stress-related burdens on children and adolescents, which could lead to improvements in their psychological complaints in adult period.
\end{abstract}

Keywords: Adverse Childhood Experience, Mental Illnesses, Prevention.

\section{INTRODUCTION}

Studies are progressively showing the significance of early life events to an individual's mental and physical health throughout life. Exposure to ACEs is common among young children and has become urgent public health issues related to adverse mental outcomes during adulthood. According to the survey of Centers for Disease Control and Prevention, the prevalence of ACE in the United States is estimated to be $60 \%$ of the population [9]. ACEs not only causes short-term or direct harm to individuals, but it also has serious adverse effects on long-term physical health and social development of individuals [18]. A case-control study in California finding that the prevalence of subsequently engaging in self-harm and violence towards other people is notably higher when young people experienced ACEs, which is harmful to social stability [1]. Previous research suggested that adults who have experienced several ACEs would get exposed to greater risks of developing health problems, such as heart disease, depression, and chronic lung disease. Thus, it is worth understanding the factors that are associated with mental illness as well as the importance of maintaining a good mental health. This article will examine the associations between specific ACEs and mental health issues and the undergoing prevention measures so that it could provide support and evidence for early detection and interventions.

\section{LITERATURE REVIEW OF ACE}

\subsection{Background: Previous Work on Adverse Childhood Experience (ACE)}

The first seminal ACE study triggered a surge of interest in the long-term consequences of childhood trauma exposure. In addition to basic research, it introduced a concise index of childhood adversity that became widely used for screening. Family dysfunction, as well as physical and mental abuse were included in the initial ACE study. Physical or emotional neglect, as well as the loss of a parent due to divorce or death were later included [8]. Some researchers are now looking into the long-term effects that ACEs have on people's health, as well as the necessity of addressing all types of stresses that can arise in children's lives rather than focusing on just one. Findings suggest that the number of ACEs and their accompanying risks for mental diseases change throughout time, with those who have at least four ACEs having a larger risk of dangerous health outcomes than those who have none [4].

Furthermore, exposure to individual ACE indicators, rather than a combination of ACE indicators, has been demonstrated in certain studies to be important. Children who experience parental violence have difficulty with language, social connections, and fine motor skills as they grow up. As a result, it's critical to examine both the total number of ACEs and the individual ACE indications 
that youngsters are exposed to. Many academics are still devoted to investigating ACEs, and they have completed numerous studies and published numerous articles. In the table 1 below, I have summarized some influential articles.

Table 1. Conclusion OF ACEs studies

\begin{tabular}{|c|c|c|c|c|c|}
\hline $\begin{array}{l}\text { Reference } \\
\text { NUMBER }\end{array}$ & Author & $\begin{array}{l}\text { Publication } \\
\text { Year }\end{array}$ & Country & study type & ACEs Type \\
\hline [2] & $\begin{array}{l}\text { Matthay } \\
\text { EC }\end{array}$ & 2018 & America & case-control & interpersonal violence \\
\hline [3] & Dube SR & 2003 & America & Retrospective cohort study & $\begin{array}{l}\text { emotional abuse, sexual abuse, emotional } \\
\text { neglect,physical } \\
\text { neglect,Household Dysfunction }\end{array}$ \\
\hline [9] & $\begin{array}{l}\text { Polanco- } \\
\text { Roman L }\end{array}$ & 2021 & America & cohort study & $\begin{array}{l}\text { child maltreatment (physical, sexual, and emotional } \\
\text { abuse and neglect), exposure to violence, parental } \\
\text { loss (separation, divorce, and death), and parental } \\
\text { maladjustment (mental health problems, substance } \\
\text { or alcohol abuse, intimate partner violence, and } \\
\text { incarceration) }\end{array}$ \\
\hline$[12]$ & Felitti VJ & 1998 & America & cohort study & $\begin{array}{l}\text { psychological, physical, sexual abuse; violence } \\
\text { against mother; living with household members, } \\
\text { mentally ill,suicidal,imprisoned }\end{array}$ \\
\hline [13] & Dube SR & 2009 & America & Retrospective cohort study & $\begin{array}{l}\text { childhood physical, emotional and sexual abuse; } \\
\text { witnessing domestic violence; growing up with } \\
\text { household substance abuse, mental illness, parental } \\
\text { divorce, and/or an incarcerated household member }\end{array}$ \\
\hline [15] & Roza SJ & 2019 & Iraq & cross-sectional study & $\begin{array}{l}\text { physical neglect, emotional neglect, physical } \\
\text { abuse,sexual abuse }\end{array}$ \\
\hline [18] & $\begin{array}{l}\text { Rytilä- } \\
\text { Mannine } \\
\text { n M }\end{array}$ & 2018 & Finland & cross-sectional study & social dysfunction, family dysfunction \\
\hline
\end{tabular}

\subsection{How ACEs cause different mental disorders}

Early emotional abuse, physical abuse, sexual abuse, emotional neglect, physical neglect, or development experiences in a dysfunctional household are all examples of ACEs. Some normal developmental processes may be disrupted as a result of ACEs' mental health effects [3], particularly regarding to critical periods of developmental tasks. For example, it would influence the process of cognitive appraisal and increasing the risk of poor adult mental disease. Due to the variability of behavioral and biological development in childhood, exposure to these ACEs can lead to toxic stress responses in children $[5,6]$, which ultimately leads to a higher risk of health and behavior problems. This change of psychosocial environments interacted by gene and environment is one of important links and influence the risk of problem behaviors and psychiatric disorders across the life span. Among Puerto Rican's young adult's cohort study [4], in comparison to young men, young women had a higher rate of lifetime suicide attempts, which were relevant to their higher exposure of ACEs experiences (e.g., physical abuse and neglect).
Based on these findings and evidence that childhood trauma affects adulthood, the author believes that people need intervention strategies to protect the mental health of people who have experienced adversity in childhood.

\subsection{Purpose: Prevention of ACEs}

Since ACE are among the highest co-occurring documented risk factors for the onset set of mental illness in adulthood, and these changes are associated with physical, mental, behavioral, and developmental damage, in recent years, a growing body of literature on ACEs have shifted from delineating how ACEs negatively affect adulthood health to preventing ACEs in children and teenagers [2]. Many children who have experienced multiple traumas are not likely to receive treatment and remediation in the traditional treatment, which makes the prevention even more urgent.

Furthermore, because the prevalence of ACEs has been high for a long time, it is critical to understand how parents' positive early life experiences can prevent ACEs from being passed down through generations. According 
to Masten et al. [7], family adversity, as well as family resilience, typically begins in previous generations.

\section{RELATIONSHIP BETWEEN ACE AND MENTAL HEALTH}

\subsection{ACE and Depression}

The association between the total number of ACEs and mental health has been studied extensively. Four or more ACEs raised the probability of depression (4.5 times) according to a survey conducted by a big US HMO [8]. Physical and emotional neglect were reported to be the most common forms of ACEs among Iraqi young adults, both of which are linked to depression in adulthood [16]. Physical and emotional maltreatment were revealed to be the most common forms of ACEs among young adults in Iraq [16], both of which are linked to depression in adulthood. Posttraumatic stress symptoms, like nightmares and flashbacks, due to ACEs will develop among young people and produce a lasting impact on behavioral and emotional development. Moreover, the accumulation of psychological stress not only affect mental health, but also correlated with nonpsychological medical conditions [10] including depression. After childhood adversity, some externalizing (e.g., violence) maladaptive behaviors are more likely to occur. As a result, children with problem behaviors are more likely to have clinically significant mental diseases and physical health difficulties later in life. Adults who were worried or sad as children were also found to be more sensitive to depression [11]. Children cannot reasonably resolve and resist the negative effects of the external environment and bad behavior, resulting in the consequences that cannot be erased.

\subsection{ACE and Anxiety}

Similar results were found for other psychological conditions, including anxiety. A study from the Dutch general population investigated behavioral and emotional problems for young children 4-16 years of age. At followup 14 years later, researchers found that those people were more likely to have anxiety disorders if they experienced childhood externalizing problem behaviors [11]. ACEs are known to cause long-term harmful effects with an increase in psychological stress. When the ability to tolerate or recover from negative emotions is weak, anxiety can be realized. It has been found that children who have had four or more ACEs are more likely to suffer from anxiety and depression than children who have not had these events [17].

\subsection{ACE and Suicidality}

Suicidality are increasing substantially from childhood into adolescence, which is an urgent problem threatens public health. However, the forming of suicidal thought may involve a variety of factors in life that are associated with thoughts and actions aimed at putting an end to one's life Suicide thoughts was shown to be prevalent in 12.1 percent of people in one study [15]. Suicide is the largest cause of mortality among the young in developed countries, accounting for $17.6 \%$ of all fatalities among those aged 15 to 29 years [15]. Suicide is the result of a complex interaction of genetic, biochemical, psychiatric, psychological, social, and cultural variables. ACEs largely influence changes in brain structure and form cognitive and affective styles to predispose mental health problems, according to the factors mediating the association between ACEs and suicidality. However, not all suicidal teens exposed to ACEs will commit suicide. When an adolescent is exposed to ACEs, some precautions can be taken to prevent suicide thoughts from developing. Psychiatric problems, impulsivity, and familial and social dysfunctions are factors that may play a potential role connected ACEs and suicidality in adolescents [14].

\section{MEASURES OF PREVENTION}

The ACE does cause some trauma to the human body and mind. However, through the research and experiments of scholars, it is found that we can take some measures at different stages to prevent such trauma or treat the trauma that has been formed. The corresponding process and measures are shown in the figure 1 below. I will also elaborate on these measures below. 


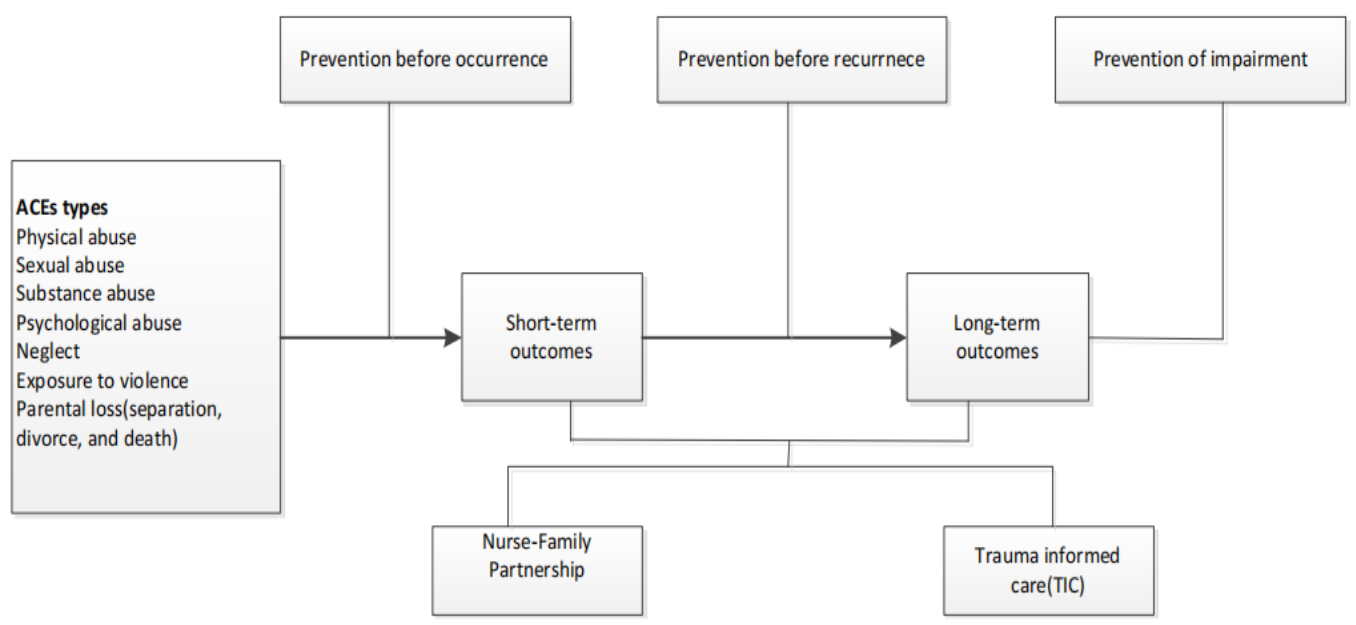

Figure 1 Prevention Phases of ACEs

\subsection{Trauma informed care (TIC)}

Individuals who experienced various adversities and resultant emotional dysregulations might present with posttraumatic stress disorder characterized with dissociation and increased automatic reactivity. The Substance Abuse and Mental Health Services Administration (SAMHSA) defines TIC as "a traumainformed program, organization, or system that recognizes and appreciates the pervasive impact of trauma." Furthermore, the SAMHSA emphasizes the six broad TIC principles of safety, trustworthiness, and transparency, peer support, collaboration, and mutuality, empowerment, voice, and choice, as well as cultural, historical, and gender problems. It implies that TIC necessitates assistance at the highest echelons of a company. TIC is founded on the idea of screening traumatized children, then screening for resiliency, family functional capacity, community-based resources, behavioral and neuropsychological tests, and past trauma interventions.

\subsection{Nurse-Family Partnership}

Pediatric nurses can identify at-risk children, provide parents with expected guidance, and recommend mental health services and community programs. Pediatric nurses can help children avoid traumatic medical events and provide resilience education and skills development. The help of pediatric nurses can greatly reduce the longterm impact of negative experiences on children's mental health.

\subsection{Primary, Secondary, Tertiary preventions}

Primary prevention, according to the public health approach, includes avoiding ACEs, which means that children are exposed to less adversity as they grow up and are less likely to produce children who are exposed to ACEs. Secondary prevention entails taking steps to mitigate the immediate and short-term effects of ACEs. Efforts to treat and decrease the long-term effects of ACEs are included in tertiary prevention. Primary prevention, on the other hand, must have the largest individual and community impact. As a result, some child abuse prevention measures, such as emergency nurseries, programs that provide referrals to services such as homeless shelters and food pantries, and greater mental health and substance addiction treatment availability, can reduce the likelihood of $\mathrm{ACE}$ occurrences at the population level.

\section{DISCUSSION}

\subsection{Study Limitations}

Despite this study tying childhood adversity to adult mental health, the paucity examining the effects of ACEs during some specific period (e.g., later adolescence and adulthood) constitutes a weak link in the developmental and epidemiological literature. Findings suggested that ACEs predicted worsening of mental health. Because this review includes many cross-sectional surveys, it is not easy to longitudinally determine the effects of ACEs on depression, anxiety, or suicidality (suicide ideation). Therefore, we cannot infer causal relationships between ACEs and mental diseases. Besides, recall bias happened because most of data collection related to retrospective reports, and we cannot ignore this effect on the final outcomes.

\subsection{Suggestions for preventive measures}

With the growing use of artificial intelligence in healthcare, research is mostly focused on cancer, nervous system disorders, and cardiovascular diseases. Researchers are gradually attempting to determine if AI 
specialized to mental health and mental disease might enhance clinical practice as a result of these applications, indicating that AI technology has great potential to transform mental healthcare [12]. These investigations exhibited remarkable accuracy and gave outstanding instances of mental disorder prediction and diagnosis. Electronic health records (EHRs), mood rating scales, brain imaging data, novel monitoring systems (e.g., smartphone, video), and social media platforms have all been used in studies of AI and mental health to predict, classify, or subgroup mental health illnesses such as schizophrenia, depression, or other psychiatric illnesses, and suicidality. Much of the research, however, should be regarded early proof-of-concept work indicating the possibility of applying machine learning (ML) algorithms [13] to address mental health concerns, and more population tests are needed to achieve the best results. Furthermore, it has the potential to help mental health professionals re-define mental diseases more objectively than DSM-5, identify these illnesses early, and personalize treatments based on a person's unique traits.

\section{CONCLUSION}

ACEs have a significant impact on adolescent mental illnesses. The public health impact of ACEs is evident throughout the early transition to adulthood, when there is a strong link between anxiety, depressive symptoms, and suicidality. This research revealed the crucial need for early adverse experience prevention and intervention programs, such as TIC and Nurse-Family Partnership, to address the mental health repercussions of early bad experiences.

\section{ACKNOWLEDGMENT}

I am grateful to all my teachers who have directly or indirectly taken part in assisting me to finish this paper. Without their support and assistance, I could not finish this paper. I would like to express my sincere thanks to Professor Chiang-shan Ray Li, who helps me a lot in topic selecting. Also, I want to thank Yuanyuan Dong for her valuable and inspiring suggestions.

\section{REFERENCES}

[1] EC. Matthay, K. Farkas, J. Skeem, J. Ahern, "Exposure to Community Violence and Self-harm in California: A Multilevel, Population-based, CaseControl Study," Epidemiology. 2018 Sep;29(5):697706. doi: 10.1097/EDE.0000000000000872. PMID: 29889134; PMCID: PMC6066408.

[2] CSSP. (2020a). "Strengthening families: Increasing positive outcomes for children and families," https://cssp.org/our-work/project/strengtheningfamilies/
[3] D. Finkelhor. "The victimization of children: a developmental perspective, "Am J Orthopsychiatry. 1995 Apr;65(2):177-93. doi: 10.1037/h0079618. PMID: 7611336 .

[4] L. Polanco-Roman, K. Alvarez, T. Corbeil, P. Scorza , M.Wall, MS. Gould , M. Alegría , H. Bird, GJ. Canino, CS. Duarte, Association of Childhood Adversities With Suicide Ideation and Attempts in Puerto Rican Young Adults. JAMA Psychiatry. 2021 May 5. doi: 10.1001/jamapsychiatry.2021.0480. Epub ahead of print. PMID: 33950163.

[5] M. Bucci , SS. Marques, NB. Harris, "Toxic Stress in Children and Adolescents," Adv Pediatr. 2016 Aug;63(1):403-28. doi: 10.1016/j.yapd.2016.04.002. PMID: 27426909.

[6] SB.Johnson , AW. Riley, DA. Granger, J.Riis, “The science of early life toxic stress for pediatric practice and advocacy,"Pediatrics. 2013 Feb;131(2):319-27. doi: 10.1542/peds.2012-0469. Epub 2013 Jan 21. PMID: 23339224; PMCID: PMC4074672.

[7] SB. Johnson, AW. Riley, DA. Granger, J. Riis, "The science of early life toxic stress for pediatric practice and advocacy," Pediatrics. 2013 Feb;131(2):319-27. doi: 10.1542/peds.2012-0469. Epub 2013 Jan 21. PMID: 23339224; PMCID: PMC4074672.

[8] VJ. Felitti, RF. Anda, D.Nordenberg, DF.Williamson, AM. Spitz, V. Edwards, MP.Koss , JS. Marks, "Relationship of childhood abuse and household dysfunction to many of the leading causes of death in adults, "The Adverse Childhood Experiences (ACE) Study. Am J Prev Med. 1998 May;14(4):245-58. doi: 10.1016/s07493797(98)00017-8. PMID: 9635069.

[9] Centers for Disease Control and Prevention (CDC). Adverse childhood experiences reported by adults -- five states, 2009. MMWR Morb Mortal Wkly Rep. 2010 Dec 17;59(49):1609-13. PMID: 21160456.

[10] SR. Dube, D. Fairweather, WS. Pearson, VJ. Felitti, RF. Anda, JB. Croft, "Cumulative childhood stress and autoimmune diseases in adults," Psychosom Med. 2009 Feb;71(2):243-50. doi: 10.1097/PSY.0b013e3181907888. Epub 2009 Feb 2. PMID: 19188532; PMCID: PMC3318917.

[11] SJ. Roza, MB. Hofstra, van der Ende J, FC.Verhulst, " Stable prediction of mood and anxiety disorders based on behavioral and emotional problems in childhood: a 14-year follow-up during childhood, adolescence, and young adulthood.,"Am J Psychiatry. 2003 Dec;160(12):2116-21. doi: 10.1176/appi.ajp.160.12.2116. PMID: 14638580. 
[12] S.Graham, C. Depp , EE. Lee, C. Nebeker, X. Tu, HC. Kim, DV. Jeste, "Artificial Intelligence for Mental Health and Mental Illnesses: an Overview," Curr Psychiatry Rep. 2019 Nov 7;21(11):116. doi: 10.1007/s11920-019-1094-0. PMID: 31701320; PMCID: PMC7274446.

[13] ABR. Shatte, DM. Hutchinson, SJ.Teague, " Machine learning in mental health: a scoping review of methods and applications," Psychol Med. 2019 Jul;49(9):1426-1448. doi: 10.1017/S0033291719000151. Epub 2019 Feb 12. PMID: 30744717.

[14] A. Kacem , Z. Hammal , M. Daoudi , J. Cohn , “ Detecting Depression Severity by Interpretable Representations of Motion Dynamics,"Proc Int Conf Autom Face Gesture Recognit. 2018 May;2018:739-745. doi: 10.1109/FG.2018.00116. Epub 2018 Jun 7. PMID: 30271308; PMCID: PMC6157749.

[15] AM. Chekroud, RJ. Zotti, Z. Shehzad, R.Gueorguieva, MK. Johnson, MH. Trivedi, TD. Cannon, JH. Krystal JH, PR. Corlett, "Cross-trial prediction of treatment outcome in depression: a machine learning approach,"Lancet Psychiatry. 2016 Mar;3(3):243-50. doi: 10.1016/S22150366(15)00471-X. Epub 2016 Jan 21. PMID: 26803397.

[16] AF. Al Shawi, YT. Sarhan, MA. Altaha, “Adverse childhood experiences and their relationship to gender and depression among young adults in Iraq: a cross-sectional study," BMC Public Health. 2019 Dec 16;19(1):1687. doi: 10.1186/s12889-019-79579. PMID: 31842837 ; PMCID: PMC6916082.

[17] T. Watt, N. Ceballos , S.Kim ,X. Pan, S. Sharma, "The Unique Nature of Depression and Anxiety among College Students with Adverse Childhood Experiences,"J Child Adolesc Trauma. 2019 Jul 2;13(2):163-172. doi: 10.1007/s40653-019-00270-4. PMID: 32549928; PMCID: PMC7289944.

[18] S.T. Lipscomb, E. Goka-Dubose, Hur, R. E. H., \& A. Henry, "Adverse childhood experiences, and instability in Children's care and Parents' work," Children and Youth Services Review, 102, 243-250. doi:

https://doi.org/10.1016/j.childyouth.2019.05.008 